\title{
Detection of humoral immune response induced in horses vaccinated with inactivated Equine Herpes Virus Vaccine
}

\author{
Mohamed Samy Abousenna ${ }^{1^{*}}$, Heba A Khafagy ${ }^{1}$, Nermeen Gouda Shafik ${ }^{1}$, Neveen Mounir Abdelmotilib², Ibrahim S. Yahia ${ }^{3,4,5}$
}

DOI. 10.21931/RB/2022.07.01.21

${ }^{1}$ Central Laboratory for Evaluation of Veterinary Biologics, Agriculture Research Center, P .0.Box 131 El-Seka El-Baida ST., Abbasia,11318, Cairo, Egypt.

${ }^{2}$ Arid Lands Cultivation Research Institute, City of Scientific Research and Technological Application, Bourg El-Arab, Alexandria, Egypt.

${ }^{3}$ Laboratory of Nano-Smart Materials for Science and Technology (LNSMST), Department of Physics, Faculty of Science, King Khalid University, Saudi Arabia.

${ }^{4}$ Research Center for Advanced Materials Science (RCAMS), King Khalid University, Abha 61413, P.0. Box 9004, Saudi Arabia.

${ }^{5}$ Nanoscience Laboratory for Environmental and Bio-medical Applications (NLEBA), Semiconductor Lab., Department of Physics, Faculty of Education, Ain Shams

University, Roxy, 11757 Cairo, Egypt.

Corresponding author: mohamedsamy2020@hotmail.com

\begin{abstract}
Equine herpesviruses (EHV1 and EHV4) are essential in horses. Repeated cases of infection and abortion in mares who have regularly vaccinated impetus us to determine to investigate the humoral immune response after post-vaccination with the same inactivated vaccine and the best vaccination protocol. Twelve healthy susceptible horses were divided into four groups (3 horse /group). The first group was vaccinated I/M with inactivated Equine Herpes (type 1 and 4), where each horse was inoculated with a single dose $(2 \mathrm{ml} /$ dose /horse). The second group was vaccinated with inactivated Equine Herpes (type 1 and 4) then a booster dose after two months. The third group was vaccinated with inactivated Equine Herpes (type 1 and 4 ) followed by two booster doses at two months intervals. Three horses were kept as a negative control in the fourth group. Serum samples were tested for the EHV and antibodies using virus Neutralization Test (VNT) and ELISA; it was found that VNT against EHV-1 indicated that the neutralizing antibody titer value $\geq 4$ fold titer rise had been demonstrated at 28th-day post-vaccination for all vaccinated horse groups, it was demonstrated that the vaccinated horse group (1) indicated the significant greater titer values compared to other vaccinated groups and showed protective titer value till the end of the experiment (6 months postvaccination), There is an agreement in titer values between ELISA and VNT tests for EHV was observed, but it could not reveal the same antibodies, where the ELISA measures antibodies against EHV1-4. It was concluded that the single-dose vaccination protocol was more appropriate for horse vaccination than other vaccination protocols.
\end{abstract}

Key words: Equine Herpesvirus, Rhinopneumonitis, Serological test, vaccine.

\section{Introduction}

Equine herpesvirus 1, 4 (EHV-1 and EHV-4) is an equine viral infectious disease that is endemic in most territories. The virus belongs to the family Herpesviridae ${ }^{1}$. It is transmitted by inhalation of aerosols of virus-laden respiratory secretions ${ }^{2}$ The disease causes respiratory illness, abortion, and occasionally neonatal mortality in horses. Abortion in mares usually occurs after two to four weeks of infection in the third trimester of the gestation period ${ }^{3}$. The morbidity rate is increased in young horses sharing the same air space. The primary infection source could be the placental fluids and aborted tissues of infected mares, where it contains a highly high payload of $E H V^{4}$. Although equine Herpesvirus disease has variable clinical manifestations, EHV-1 is a significant cause of neurological disease in horses ${ }^{5}$. Vaccination is an essential tool in disease control. The available commercial vaccines have two antigen content found in inactivated form for prevention and protection from respiratory illness and abortion due to infection, but there is no instruction or evidence about vaccine efficacy to prevent the neurologic form ${ }^{6}$. Although horses were vaccinated with inactivated Equine Herpes virus vaccine, a repeated cases of abortion occurred. So this study prompted us to investigate the immune response duration post-vaccination with inactivated Equine Herpesvirus vaccine and the most suitable time for booster dose to determine the best vaccination protocol. Serum antibody levels against EHV 1-4could be screened by virus neutralization test $(\mathrm{VNT})^{7}$ and ELISA ${ }^{8}$.

\section{Materials and methods}

\section{Virus}

A freeze-dried local strain of EHV type 1 was used for the virus-neutralizing test. It was obtained from Equine Vaccine Research Dept., Veterinary Serum and Vaccine Research Institute (VSVRI), Abbassia, Cairo, according to Magda ${ }^{9}$.

\section{Tissue culture}

African green monkey kidney cells (Vero) were obtained from the Foreign Animal Disease Diagnostic Laboratory (FADDL), Plum Island, USA, and used for virus propagation and virus neutralization test (VNT) 


\section{Inactivated Equine Herpesvirus Vaccine}

The local commercial inactivated Equine Herpesvirus (type 1 and 4) was used in this study had been evaluated and approved before by Central Laboratory for Evaluation of Veterinary Biologics, (CLEVB).

\section{Animals and Vaccination Protocols}

Twelve healthy susceptible horses, between 6 and 10 years old, with low neutralizing antibody titers (< Log10 0.6 TCID50) against $E H V-1^{10}$ ) were divided into four groups ( 3 horse /group). The first group was vaccinated I/M with inactivated EHV1-4 vaccine, where each horse was inoculated with a single dose $(2 \mathrm{ml} /$ dose /horse). The second group was vaccinated with inactivated EHV1-4 vaccine then a booster dose after two months. The third group was vaccinated with inactivated EHV1-4 vaccine followed by two booster doses at two months intervals. Three horses were kept as a negative control in the fourth group.

All animal groups were kept in a designated area for CLEVB at the animal facility house of the government veterinary hospital, Abbasia, Cairo, Egypt.

\section{Evaluation of Humoral immune response}

\section{Virus Neutralization Test (VNT)}

Serum samples were tested for the EHV-1 and antibodies using the virus Neutralization Test (VNT) and expressed as a neutralizing index according to Senthil and Parames ${ }^{11}$.

\section{ELISA Kit}

Equine Herpesvirus type 1 and 4 Differentiating test Svanova lot, A69377- REF, 10-3100-02. It was supplied by

\begin{tabular}{|c|c|c|c|c|c|}
\hline Time of Sampling & Group 1 & Group 2 & Group 3 & Group 4 (control) \\
\hline Zero day & 0.3 & 0.2 & 0.3 & 0.3 \\
\hline \multicolumn{5}{|c|}{ Vaccination (2ml/ dose /horse) } \\
\hline $\mathbf{1 4}^{\text {th }} \mathbf{D P V}$ & 1.2 & 0.9 & 1.14 & 0.3 \\
\hline $\mathbf{2 8}^{\text {th }} \mathbf{D P V}$ & 1.8 & 1.5 & 1.68 & 0.6 \\
\hline $\mathbf{2 M P V}$ & 2.04 & 1.92 & 2.04 & 0.4 \\
\hline 3MPV & 2.4 & 2.04 & 2.1 & 0.3 \\
\hline 4MPV & 2.28 & 1.8 & 1.86 & 0.5 \\
\hline & & Booster dose & \\
\hline 5MPV & 1.92 & 1.68 & 1.44 & 0.5 \\
\hline 6MPV & 1.8 & 1.5 & 1.08 & 0.4 \\
\hline
\end{tabular}

DPV: Days post-vaccination - MPV: Month's post-vaccination.

Response rate $\geq \mathbf{4}$ fold titer rise

Group 1: EHV inactivated single vaccine dose.

Group 2: EHV inactivated vaccine with a booster after two months

Group 3: EHV inactivated vaccine with two booster doses two months interval

Control: Negative control without injection the Central Laboratory for Evaluation of Veterinary Biologics (CLEVB). The antibody values were measured at a $450 \mathrm{~nm}$ absorbance reading of each well. According to the manufacturer's instructions, positive values were considered from a cut off 0.2 .

\section{Results}

Table 1 shows the VNT results against EHV-1 for the vaccinated horse groups (1,2 and 3) with inactivated EHV (1 and 4 ) vaccine, all vaccinated horse groups indicated an increase in titer values at 3 months post-vaccination, the groups ( 2 and 3) showed a slight decrease in antibody titer values after 2 nd dose of vaccination compared to group (1). In contrast, the 3rd dose of vaccination in group (3) indicated a decrease in antibody titer values below the protective value ( $\geq 4$ fold titer rise) compared to groups ( 1 and2), which was demonstrated at 5 months post-vaccination. The vaccination protocol was used in the group (1) to afford high antibody titer compared to other vaccination protocols.

Table 2 shows the ELISA results against EHV 1 and 4 for the vaccinated horse groups (1,2 and 3 ) with inactivated EHV (1 and 4) vaccine, all vaccinated horse groups indicated an increase in mean ELISA values at 2 months post-vaccination, the groups (2 and 3 ) showed a decrease in mean ELISA values after 2 nd dose of vaccination compared to group (1). In contrast, the 3 rd dose of vaccination in group (3) indicated a decrease in mean ELISA values similar to group (2) mean ELISA values, demonstrated at 5 months post-vaccination. The vaccination protocol was used in the group (1) to afford high antibody titer compared to other vaccination protocols.
Table 1. Virus neutralizing antibody titer in vaccinated horses with inactivated Equine Herpes virus vaccine. 


\begin{tabular}{|c|c|c|c|c|}
\hline Time of Sampling & Group 1 & Group 2 & Group 3 & Group 4 (control) \\
\hline Zero day & 0.25 & 0.19 & 0.23 & 0.2 \\
\hline \multicolumn{5}{|c|}{ Vaccination (2ml/ dose /horse) } \\
\hline 14th DPV & 0.89 & 0.78 & 0.88 & 0.18 \\
\hline 28thDPV & 1.28 & 1.19 & 1.22 & 0.21 \\
\hline 2MPV & 1.53 & 1.45 & 1.58 & 0.22 \\
\hline \multicolumn{5}{|c|}{ Booster dose } \\
\hline 3MPV & 1.29 & 0.9 & 0.96 & 0.22 \\
\hline 4MPV & 0.85 & 0.56 & 0.67 & 0.23 \\
\hline \multicolumn{5}{|c|}{ Booster dose } \\
\hline 5MPV & 0.76 & 0.33 & 0.25 & 0.21 \\
\hline 6MPV & 0.65 & 0.35 & 0.17 & 0.22 \\
\hline
\end{tabular}

DPV: Days post-vaccination - MPV: Month's post-vaccination.

Group 1: EHV inactivated single vaccine dose.

Group 2: EHV inactivated vaccine with a booster after two months.

Group 3: EHV inactivated vaccine with two booster doses two months interval

Control: Negative control without injection

Table 2. Seroconversion of horses vaccinated with inactivated Equine Herpes virus vaccine tested by ELISA.

\section{Discussion}

Hygienic measures and vaccination control EHV1 and EHV4 infections. Commercial vaccines, either inactivated or modified live virus (MLV), have been shown to afford protection for vaccinated animals, lessening EHV-related respiratory disease, neurological disease, and abortion under experimental conditions ${ }^{12}$. Many studies have demonstrated the humoral responses of on-field vaccinated horses using serological assays ${ }^{13}$.

This study compared different vaccination protocols using inactivated EHV1-4 vaccine, and the humoral immune responses were screened post-vaccination to demonstrate the best vaccination protocol using VNT and ELISA.

The VNT was carried out for detection of neutralizing antibody against EHV-1 for serum samples of vaccinated horse groups (1,2 and 3) within 6 months post- vaccination, the results of VNT indicated that the neutralizing antibody titer value $\geq 4$ fold titer rise (the response rate. (4)) has been demonstrated at 28th day post vaccination for all vaccinated horse groups, it was demonstrated that the vaccinated horse group (1) indicated greater titer values compared to other vaccinated groups and showed protective titer value till the end of the experiment (6 months post vaccination), while the vaccinated horse groups ( 2 and 3 ) indicated apparent decrease in antibody titer values after 2 nd dose of vaccination, furthermore subsequent decrease in antibody titer values was demonstrated after 3rd dose of vaccination in vaccinated horse group(3), as shown in table No.(1), interestingly, similar study demonstra- ted that horses received three doses of inactivated EHV-Ivaccine, a month apart, the Geometric mean (GM) titer increased to the response rate after the first dose, but no evident titer rises were observed after the second and third doses ${ }^{14}$.

There is an agreement in titer values between ELISA and VNT tests for EHV was observed, but it could not reveal the same antibodies, where the ELISA measure antibodies against EHV1-4 as shown in table No. (2) while VNT measure antibodies against EHVI only, a similar study demonstrated it was observed no correlation in the antibody titer values between ELISA and SN tests for EHVI. This could be justified that the $\mathrm{SN}$ test measured the antibodies differ from those measured by ELISA ${ }^{15}$.

The obtained results of our study showed a clear difference in immune response between three vaccination protocols which was explained by other studies, the immune response was not observed post-vaccination-especially the effect of booster dose vaccination. This failure of the humoral immune response probably due to the type of vaccine, a study used a modified live vaccine (MLV) and an inactivated vaccine, the serum neutralization (SN) assays indicated high SN titer values in MLV vaccinated mares when compared to those vaccinated with the inactivated vaccine, this could be explained that the antibody response is probably vaccine-type dependent ${ }^{13-16}$ in another study, the hypothesis of the decrease in antibody titer after the first inoculation of the vaccine has a neutralizing action could be accepted but it does not work or justify 
the decrease of antibody titer values after subsequent ones of vaccination. This reduction of antibody titer values followed by subsequent ones could be explained by the antibodies interference hypothesis due to the presence of a high titer of anti-EHV4 antibodies which has an antigenic correlation with EHV117. In another study showed that the failure of immune response had a different hypothesis, it could be a different antigen structure between vaccinal strain and the virus used in VNT, and inappropriate vaccine formulation, or by the high antibody titer before vaccination ${ }^{15}$

\section{Conclusions}

It was concluded that the single-dose vaccination protocol was more appropriate for horse vaccination than other vaccination protocols, thus affording higher antibody titers, longer immunity duration and economic wise; therefore, further investigation is desperately needed to assess the cellular immune response post-vaccination and its role in horse protection.

\section{Author Contribution}

Experiments were designed by NGS and MSA; the experiments were performed HAK, NMA and MSA. NMA accomplished data analysis. ISY and MSA. HAK and MSA wrote the manuscript.

\section{Institutional Review Board Statement}

Institutional Animal Care and Use Committee at Central Laboratory for Evaluation of Veterinary Biologics approved the research manuscript, and it has been reviewed under our research authority and is fulfilling bioethical standards.

\section{Data Availability Statement}

All data generated or analyzed during this study are included in this published article.

\section{Acknowledgments}

The authors express their appreciation to the Deanship of Scientific Research at King Khalid University for funding this work through the research groups program under grant number R.G.P.2/111/41. The authors extend their appreciation to the Deputyship for Research and Innovation, Ministry of Education, in Saudi Arabia, for funding this research work through the project number: (IFP-KKU-2020/9).

\section{Conflicts of Interest}

The authors declare no conflict of interest.

\section{Bibliographic references}

1. ICTV Taxonomy (html). International Committee on Taxonomy of Viruses (ICTV). Retrieved 17 January (2019). https://talk.ictvonline.org/.

2. Goodman, L.B.; Loregian, A.; Perkins, G.A.; Nugent, J.; Buckles, E.L.; Mercorelli, B.; Kydd, J.H.; Palu, G.; Smith, K.C.; Osterrieder, N. \& Davis-Poynter, N.A point mutation in a herpesvirus polymerase determines neuropathogenicity. PLoS Pathogen.2007, 3 (11), e160.

3. Carter, G.R.; Flores, E.F.; Wise, D.J."Herpesviridae". A Concise Review of Veterinary Virology. Retrieved 2006-06-08.

4. OIE. Organization for Animal Health. Equine rhinopneumonitisInfection with equine herpesvirus-1 and -4. En: OIE. World Organization for Animal Health. Manual of diagnostic tests and vaccines for terrestrial animals (mammals, birds, bees). Paris:OIE;
2018,Chapter 3.5.9. p 1320-1332

5. Goehring, L.S.; van Winden, S.C.; van Maanen, C.; Sloet vaOldruitenborgh-Oosterbaan, M.M. Equine herpesvirus typel-associated myeloencephalopathy in The Netherlands: a four-year retrospective study (1999 -2003). J. Vet. Intern. Med. 2006, 20:601-607.

6. Laura, B.; Goodman, A.B.; Christine, W.A.; Edward, J.; Dubovi, A.B.; Carvel, G.A.B. and Bettina, W.A.B. Immunological Correlates of Vaccination and Infection for Equine Herpesvirus 1, Clinical and Vaccine Immunology 2011,p. 235-241

7. Thomson GR, Mumford JA, Campbell J, Griffiths $L$ and Clapham P. Serological detection of equid herpesvirus 1 infections of the respiratory tract. Equine Vet. J.2011, 8, 58-65.

8. Crabb, B.S. and Studdert, M.J. Equine herpesviruses 4 (equine rhinopneumonitis virus) and 1 (equine abortion virus). Adv. Virus Res. 1995, 45, 153-190.

9. Magda, A.K.; Maysa, H.; Safaa, A.W.; Nehal, S.S.; Nashwa, K.M.; Heba, E.; Sohair, E. and Eman, E. Prospective studies of equine herpesvirus-1 myelo encephalo pathy in Egypt 2012 "IPPo logia, Anno24,n.3-4 settembre- dicembre 2013, p. (25-31).

10.Charles, W.; Purdy, D.; Shirley, J.; Williams, F. and Grant, B.S. Equin rhinopneumonitis virus (Herpes virus type1) Attenuation in stable monkey cell line Am. j. Vet. Res. 1977, 38 (8):1211- 1215.

11. Senthil, N.R. and Parames, W. Seroprevalence of equine herpes virus (EHV-1) in equidae using serum neutralization test. J. of Res Agric Animal Sci.2014, 2 ( 5):04-06

12. Heldens, J.G.M.; Hannant, D.; Cullinane, A.A.; Prendergast, M.J.; Mumford, J.A.; Nelly, M.; Kydd, J.H.; Weststrate, M.W. and Van den Hoven, R. Clinical and virological evaluation of the efficacy of an inactivated EHV1 and EHV4 whole virus vaccine (Duvaxyn EHV1,4). Vaccination/challenge experiments in foals and pregnant mares. Vaccine 2001, 19, 4307-4317.

13. Bresgen C, Lämmer M, Wagner B, Osterrieder N, Damiani AM Serological responses and clinical outcome after vaccination of mares and foals with equine herpesvirus type 1 and 4 (EHV- 1 and EHV-4) vaccines. Vet. Microbiol. 2012, 160, 9-16.

14. Bannai, H.; Tsujimura, K.; Nemoto, M. et al. Epizootiological investigation of equine herpesvirus type 1 infection among Japanese racehorses before and after the replacement of an inactivated vaccine with a modified live vaccine. BMC Vet Res 15, 280 (2019). https://doi.org/10.1186/s12917-019-2036-0

15. Attili, A.R.; Colognato, R.; Preziuso, S.; Moriconi, M.; Valentini, S.; Petrini, S.; De Mia, G.M.; Cuteri, V. Evaluation of Three Different Vaccination Protocols against EHV1/EHV4 Infection in Mares: Double Blind, Randomized Clinical Trial. Vaccines. 2020; 8(2):268. https://doi.org/10.3390/vaccines8020268.

16. Mumford, E.L.; Traub-Dargatz, J.L.; Carman, J.; Callan, R.J.; Collins, J.K.; Goltz, K.L.; Romm, S.R.; Tarr, S.F.; Salman, M.D. Occurrence of infectious upper respiratory tract disease and response to vaccination in horses on six sentinel premises in northern Colorado. Equine Vet. J. 2003, 35, 72-77.

17. Foote, C.E.; Love, D.N.; Gilkerson, J.R. and Whalley, J.M. Serological responses of mares and weanlings following vaccination with an inactivated whole virus equine herpesvirus 1 and equine herpesvirus 4 vaccine. Vet. Microbiol. 2002, $88,13-25$. 